\title{
Nonadherence to immunosuppression: challenges and solutions
}

\author{
This article was published in the following Dove Press journal: \\ Transplant Research and Risk Management \\ 3 June 2015 \\ Number of times this article has been viewed
}

\author{
Francesc Moreso' \\ Irina B Torres' \\ Gemma Costa-Requena ${ }^{2}$ \\ Daniel Serón' \\ 'Nephrology Department, ${ }^{2}$ Psychiatry \\ Department, Hospital Universitari \\ Vall d'Hebron, Universitat Autònoma \\ de Barcelona, Passeig Vall d'Hebron, \\ Barcelona, Spain
}

\begin{abstract}
Nonadherence to immunosuppressant treatment is common after renal transplantation involving $>20 \%$ of patients. It is associated with cellular rejection, appearance of donor-specific antibodies, and chronic rejection. It has been estimated that nonadherence can be detected in approximately $50 \%$ of failing grafts. Since the evaluation of sociodemographic factors do not allow characterizing the target population, it is necessary to combine different measures of adherence (self-reporting and collateral reporting, pill counts, biological monitoring of blood samples, or others) to increase its diagnostic accuracy. During the last decade, it has been shown that the implementation of a multidimensional intervention including information, motivation, and behavioral interventions may lead to an improvement of adherence to treatment. On the other hand, it has been shown that one-off feedback from a nurse, simplification of treatment, or financial assistance programs offered little improvement. Thus, increasing the effectiveness of adherence interventions might have a far greater impact on the long-term outcome of renal transplants than any improvement in specific medical treatments. This will require coordinated action from health professionals, researchers, health planners, and policy makers.
\end{abstract}

Keywords: renal transplantation, nonadherence, immunosuppressive treatment

\section{Introduction}

Nonadherence (NA) to treatment in acute and, especially, in chronic illnesses is a common situation in different areas of medicine. In the document published by the World Health Organization (WHO) on adherence to long-term therapies, the first take-home message states that

poor adherence to treatment of chronic diseases is a worldwide problem of striking magnitude since adherence to long-term therapy for chronic illnesses in developed countries averages $50 \%$ and, in developing countries, the rates are even lower. ${ }^{1}$

In this document, adherence was defined as "the extent to which a person's behaviour - taking medication, following a diet, and/or executing lifestyle changes, corresponds with agreed recommendations from a health care provider". Furthermore, strong emphasis was placed on the need to differentiate adherence from compliance. The main difference is that adherence requires the patient's agreement to the recommendations since patients should be active partners with health professionals in their own care. In the Non-adherence Consensus Conference Summary Report in transplanted patients published in $2009,{ }^{2}$ the following definitions were considered: compliance is "the extent to which the patient's behavior matches the prescriber's recommendation"; adherence is "the extent to which the patient's behavior matches the agreed upon prescriber's
Correspondence: Francesc Moreso Nephrology Department, Hospital Universitari Vall d'Hebron, Universitat Autònoma de Barcelona, Passeig Vall d'Hebron II 9-129, 08035 Barcelona, Spain

Tel +34932746079

Email fjmoreso@vhebron.net
Transplant Research and Risk Management 20I5:7 27-34 
recommendations"; and concordance is "an agreement reached after negotiation between a patient and a healthcare professional that respects the beliefs and wishes of a patient in determining whether, when and how medicines need to be taken". In this document, it was also agreed that the preferred term was "adherence" including collaboration between the patient and healthcare professionals.

Renal transplantation is the best treatment for end-stage renal disease because it is associated with longer survival of patients and higher quality of life than dialysis therapies. ${ }^{3}$ However, to maintain graft function, it is necessary to receive long-term immunosuppressive treatment in order to avoid acute/chronic rejection, because tolerance (ie, maintaining graft function without immunosuppression) is an exceptional situation in renal transplant recipients. Despite that adherence to immunosuppression has been the focus of interest of the transplant community over the past decades, the true prevalence of NA in different populations is not well established and a broad range of NA has been reported (from $0 \%$ to $68 \%$ ). Additionally, main risk factors for NA have not been clearly elucidated and contradictory results have been reported about the risk associated with different demographic and psychosocial characteristics. ${ }^{4}$ Thus, it is necessary to characterize the clinical epidemiology of NA to immunosuppression (rate and risk factors) in order to develop interventions to promote adherence that are cost-effective and have a reasonable chance of success. In this paper, we review different methods to monitor adherence, the current knowledge on the epidemiology of NA to immunosuppressive treatment in renal transplants, and different strategies to promote adherence to immunosuppressive drugs.

\section{Methods to monitor adherence to immunosuppressive treatment}

The estimation of the true prevalence of NA to immunosuppression in renal transplants is a difficult task, because different definitions have been employed to consider a patient as nonadherent and different methods have been used to evaluate NA. The evaluation of NA to treatment should include an assessment of the four dimensions of adherence: taking, timing, dosing, and drug holidays. ${ }^{1}$ It is important to remark that in contrast to other chronically ill patient populations, even minor deviations from the prescribed regimen (ie, taking $<98 \%$ of the tablets, taking drug holidays, or variability in timing of medication intake of $>2 \mathrm{hr}$ ) have been associated with an increased risk of late acute rejection, graft loss, and poor kidney function. ${ }^{2}$ In many studies, the definition of NA was based on missing, forgetting, or altering a dose at least once per month, ${ }^{5}$ this means that the patient misses at least 3\% of doses. However, other authors have used highest thresholds to define NA ranging from $10 \%$ to $20 \%$ of missing doses. ${ }^{6,7}$ Additionally, the timing of doses has also been considered, and in some studies, taking medication 2 hours or 2.5 hours later at least once per month has also been used to define NA. ${ }^{8}$ Nevertheless, to assess adherence to treatment as a dichotomous variable (yes/no) has limitations since NA should be considered as a continuous variable.

Assessment methods of NA can be classified as direct and indirect with advantages and disadvantages for each one (Table 1) offering different levels of sensitivity. ${ }^{9}$ Direct measures include observation of medication intake and biological assays to measure drug levels or drug metabolites in the blood or urine. Observation verifies adherence but requires direct patient-clinician encounters and it is a nonfeasible approach to monitor chronic treatments. Drug monitoring reflects the patient's consumption of immunosuppressive drugs, but results can be influenced by the half-life of drugs, metabolic rates, and white coat adherence (ie, greater adherence before a clinical visit).

Indirect measures include patient self-reporting (surveys, questionnaires, and interviews), collateral reports from family members or clinicians, prescription fills, pill counts, and electronic monitoring. Self-reporting is inexpensive and feasible in most clinical settings, but it may be biased by honesty of the patient and his/her capacity to recall. Despite its limitations, it has been one of the most commonly used methods to assess

Table I Evaluation of different methods to assess nonadherence to immunosuppression

\begin{tabular}{|c|c|c|}
\hline Method & Advantages & Disadvantages \\
\hline \multicolumn{3}{|l|}{ Direct } \\
\hline $\begin{array}{l}\text { Medication intake } \\
\text { observation }\end{array}$ & Verifies adherence & Clinically unpractical \\
\hline $\begin{array}{l}\text { Biological monitoring } \\
\text { of drug levels in } \\
\text { blood/urine }\end{array}$ & $\begin{array}{l}\text { Reflects degree of } \\
\text { immunosuppression. } \\
\text { Objective }\end{array}$ & $\begin{array}{l}\text { Drug's half-life, } \\
\text { metabolic rate, white } \\
\text { coat adherence }\end{array}$ \\
\hline \multicolumn{3}{|l|}{ Indirect } \\
\hline Self-reporting & Simple, cheap & $\begin{array}{l}\text { Bias (patient honesty, } \\
\text { recall) }\end{array}$ \\
\hline Collateral reporting & Simple, cheap & $\begin{array}{l}\text { Bias (clinicians' } \\
\text { overestimation) }\end{array}$ \\
\hline Pharmacy refill & Objective, cheap & $\begin{array}{l}\text { Not equivalent to } \\
\text { ingestion of medication } \\
\text { Requires a closed } \\
\text { pharmacy system }\end{array}$ \\
\hline Pill counts & $\begin{array}{l}\text { Objective, simple, } \\
\text { cheap }\end{array}$ & $\begin{array}{l}\text { Discarded or hoarded } \\
\text { doses }\end{array}$ \\
\hline Electronic & Provides insight & Expensive \\
\hline $\begin{array}{l}\text { monitoring gold } \\
\text { standard }\end{array}$ & $\begin{array}{l}\text { into patterns of } \\
\text { adherence (day, time) }\end{array}$ & $\begin{array}{l}\text { Discarded or hoarded } \\
\text { doses }\end{array}$ \\
\hline
\end{tabular}


adherence and there are different validated questionnaires for this purpose. One of the simplest validated scales to evaluate adherence to treatment is the Morisky Medication-taking Adherence Scale (MMAS, four-item scale), which scores as a dichotomous variable (no/yes) four questions about taking medication. ${ }^{10}$ In 2005, the Immunosuppressive Therapy Adherence Scale $^{\odot}\left(\right.$ ITAS $\left.^{\odot}\right)$ was the first validated scale to evaluate adherence in transplant recipients. ${ }^{11}$ The ITAS is also a four-item measure in which transplant recipients indicate the frequencies of the following immunosuppressive therapy adherence-related behaviors in the previous 3 months: forgetfulness to take medications, carelessness regarding medication taking, cessation of medication taking due to feeling worse, and neglect of medication taking for many reasons. Response options on a four-point Likert scale were as follows: $3=0 \%$ (none of the time), $2=1 \%-20 \%$, $1=21 \%-50 \%$, and $0 \geq 51 \%$ of the time. Item responses were summed with possible total scores ranging from 0 to 12 . Among the different questionnaires available to evaluate adherence and based on a European survey and a systematic literature review, the Transplant360 Task Force identified three self-report NA measurement tools that could be adapted to transplant clinical practice: the Basel Assessment of Adherence to Immunosuppressive Medications Scale (BAASIS ${ }^{\circledR}$ ), the Medication Adherence Self-Report Inventory, and the Brief Antiretroviral Adherence Index Questionnaire. All these instruments evaluate both the drug taking and the regularity of medication intake and are considered easy to use and score. ${ }^{12}$ The BAASIS interview ${ }^{13}$ has been widely used in renal transplants and it measures all dimensions of NA (taking, timing, dosing, and drug holidays). In this interview, participants were asked in a nonthreatening, nonjudgmental manner about how often, over the last 4 weeks, they i) had not taken their drugs (taking dimension), ii) had taken their medication $>2$ hours before or after their prescribed taking time (timing dimension), iii) had skipped at least two consecutive doses of their drugs (drug holidays), and iv) had reduced the prescribed amount of their medication (dose reduction). Responses were given on a 6-point scale ranging from 0 (never) to 5 (every day). NA was defined as any self-reported NA (response score 1-5) on any of the four items. This questionnaire has been validated in different languages..$^{14}$

The fill frequency of prescriptions requires complete pharmacy records and can be problematic when patients use a number of non-networked pharmacies. Different approaches for examining compliance using insurance claims electronically submitted to obtain reimbursement for dispensed medications have been used and included fixed time point, gaps in prescription filling, and medication possession ratio. Obviously, medication possession is not equivalent to ingestion of medication and does not assure adherence to treatment. Pill counts are easy to perform but invalidated when patients discard drugs.

In summary, state-of-the-art measures such as selfreporting, collateral reporting, prescription refills, and pill count usually under-represent NA, while providing little information about individual medication-related behavior. Electronic monitoring has been used as the reference standard to evaluate NA. It involves fitting a pill bottle with a timer/ counter, which continuously records the date and time of every cap opening. However, electronic monitoring can be used incorrectly, negatively impact established adherence routines, and even improve normal adherence through an intervention effect. In the market, there are different devices such as the Medication Event Monitoring System (MEMS) and the Helping Hand Data Capturing. The MEMS is currently regarded as the gold standard to measure adherence in clinical trials and it has been used as such in a wide range of studies on adherence to antihypertensive and lipid-lowering therapy, therapy for neurologic and psychiatric disorders, post-transplantation immunosuppressive therapy, and antiretroviral therapy. However, it has some drawbacks: it may lead to practical (eg, the pill bottle is rather large) or confidentiality issues. Besides, based on the safety regulations from the pharmaceutical companies, immunosuppressants need to stay in the blister until actual ingestion to avoid changes in stability of the drugs. Thus, in order to fit in the electronic medication container, the blister needs to be cut in individual packaged pills. The Helping Hand is an electronic monitoring tool with similar functions compared to the MEMS, but suitable for blister packages. Additionally, this device can contribute to cover privacy problems because it is smaller and easy to hide.

In summary, no single measurement strategy has been deemed optimal and a multi-method approach that combines feasible self-reporting and reasonable objective measures is the current state of the art in the measurement of adherence behavior. In this sense, in a cross-sectional study that included a sample of 249 adult kidney transplant recipients, NA was assessed using electronic monitoring over 3 months (as the reference standard) and some of the above-mentioned methods. The authors also constructed a composite adherence score including blood assays, self-reports, and collateral reports. The prevalence of NA across the measurement methods was $17.3 \%$ for electronic monitoring, $33 \%$ for biological 
assays, $12.4 \%$ for self-report, $24.9 \%$ for collateral reports, and $38.9 \%$ for the composite adherence score. The collateral reports and composite adherence score showed the lowest and highest sensitivities to NA (15.8\% and $72.1 \%$, respectively). Specificity was highest for collateral reports of at least three clinicians (93.1\%). Thus, combining measures increased diagnostic accuracy, indicating the relevance of combined measures for clinical and research purposes. ${ }^{9}$

\section{Prevalence of NA to immunosuppressive treatment}

Taking into consideration the limitations associated with different methods to assess NA and the different definitions employed across studies, in 2004 a systematic review on the frequency and impact of NA to immunosuppressive drugs after kidney transplantation ${ }^{5}$ estimated that the prevalence was $22 \%$ (interquartile range 18\%-26\%) for cross-sectional studies $(n=15)$ and 15\% (5\%-19\%) for cohort studies $(n=10)$. Later on, in 2007, a total of 147 studies published between 1981 and 2005 on kidney, heart, liver, pancreas, and lung transplants were included in a meta-analysis. ${ }^{4}$ In this report, the largest proportion of studies focused on kidney transplants (49\%) and included a total of 29,000 patients contributing to an observation of 88,000 persons per year. Across all types of transplants, the average NA to immunosuppressants was 19-25 cases per 100 persons per year. Rates varied significantly by transplant type being the highest in kidney transplant recipients (36 cases per 100 persons per year) and, interestingly, the prevalence in North America was much higher than that in Europe (33.4 vs 13.5 per 100 persons per year).

\section{NA to immunosuppression and graft failure}

The requirement for continuous immunosuppressive treatment to avoid graft rejection is well known from the beginning of the transplantation history. ${ }^{15,16}$ For example, electronic monitoring was used to evaluate azathioprine adherence during a 6-month period in 134 renal transplant recipients and this study showed a significant correlation between degree of adherence and rejection-free survival in the first 6 months post transplant. ${ }^{17}$ Lower compliance rates during the first 6 months were associated in a "dose-response" fashion with acute rejection and allograft loss. Declining compliance during the first 90 days was a strong risk factor for later acute rejection (odds ratio $=13.9$ ) and allograft loss (odds ratio $=4.3$ ). These data indicate that NA appears early posttransplant and tends to increase over time. In the meta-analysis reported in $2004,{ }^{5}$ all cohort studies showed an increased risk of graft failure in nonadherent patients. Even with the use of measures generally accepted to lack sensitivity for the detection of NA, the odds of graft loss are increased about sevenfold in nonadherent patients when compared with adherent patients.

The link between chronic rejection, graft failure, and NA was well established in the observational study conducted by the Edmonton group including 315 transplant recipients who underwent a graft biopsy for cause. During follow up, 60 patients experienced graft failure. ${ }^{18}$ In this study, NA was only recorded retrospectively by medical chart review, based on records of patient admission or strong clinical suspicion by the attending clinicians. Concerns about NA were recorded 10 times more frequently in patients whose graft subsequently failed (32\%) than in those whose grafts have not failed (3\%), and nonadherent patients had donor-specific antibodies more often than did adherent patients. Concerns about NA were recorded by the attending clinicians in 26 patients, of whom 19 experienced kidney failure, 17 of which were due to rejection ( $47 \%$ of rejection-related failures). The majority of kidney failures were attributed to antibody-mediated rejection or mixed rejection, with NA recorded in nearly half. Finally, there was evidence of antibody-mediated rejection in the majority of NA patients whose graft failed. Similar results were recently reported by the Miami group. ${ }^{19}$ In their prospectively followed cohort of 628 adult, primary kidney-alone transplant recipients with long-term follow up, the observed percentage who developed death-censored graft failure was 78.4\% (29/37) among noncompliant patients versus only $7.8 \%$ (46/591) among compliant patients. Furthermore, graft failure related with NA represented 48.1\% (26/54) of deathcensored graft failures beyond 24 months.

However, prospective studies evaluating the relationship between NA and clinical outcomes are scarce making it difficult to reach consensus on how much NA is enough to result in deleterious clinical outcomes. Scarce data have indicated that even minor deviations from the prescribed regimen (ie, $<95 \%$ adherence to the immunosuppressive regimen) are sufficient to result in poor outcomes. ${ }^{2}$ This indicates that, in contrast to other chronic diseases such as arterial hypertension or dyslipidemia, in renal transplants, minimal NA to treatment may be sufficient to increase the risk of transplant failure.

\section{Risk factors for NA to immunosuppressive treatment}

In order to apply strategies to improve adherence to immunosuppressants, it would be desirable to characterize the 
target population with the highest risk. However, this is an unrealistic approach since adherence to treatment is a dynamic process that should be monitored persistently during follow up and there are many variables that will continue to change during the transplant life. Classically, risk factors for NA can be categorized into five interrelated areas: 1) socioeconomic; 2) patient-related; 3) disease-related; 4) treatment-related; and 5) factors related to the healthcare setting and provider. Despite the absence of consistency across different studies, in Table 2 variables associated with NA to immunosuppressants in adults are summarized. It is important to remark that in the meta-analysis conducted in 2007 by Dew et al, ${ }^{4}$ demographics (male sex, younger age, and nonwhite ethnicity), education, social support, and perceived health showed little correlation with NA. The authors concluded that patients at risk for NA cannot be identified on the basis of personal characteristics but this fact should not be interpreted as a limitation, since these findings may open the door to focus on provider-related and health-system-related factors that may be more important determinants of patient adherence to medication. In this sense, the adherence rate was compared to immunosuppression using similar methodology in the US and Europe. A total

Table 2 Risk factors associated with nonadherence to immunosuppression

\begin{tabular}{|c|c|}
\hline $\begin{array}{l}\text { Areas related with } \\
\text { nonadherence }\end{array}$ & $\begin{array}{l}\text { Factors related with } \\
\text { nonadherence }\end{array}$ \\
\hline Socioeconomic & $\begin{array}{l}\text { Socioeconomic status } \\
\text { Race/culture } \\
\text { Poor social support } \\
\text { Poor social functioning } \\
\text { Illiteracy }\end{array}$ \\
\hline Patient related & $\begin{array}{l}\text { History of nonadherence } \\
\text { Adolescence } \\
\text { Psychological disorders (depression) } \\
\text { Psychiatric illness } \\
\text { Personality disorders } \\
\text { Cognitive impairment } \\
\text { Substance abuse } \\
\text { Negative beliefs in medications } \\
\text { Low conscientiousness }\end{array}$ \\
\hline Disease related & $\begin{array}{l}\text { Longer illness duration, poor disease } \\
\text { knowledge/insight }\end{array}$ \\
\hline Treatment related & $\begin{array}{l}\text { Medication side effects } \\
\text { Complex regimen } \\
\text { Lack of medication knowledge } \\
\text { No pill box/reminder system }\end{array}$ \\
\hline $\begin{array}{l}\text { Factors related to the healthcare } \\
\text { setting and provider }\end{array}$ & $\begin{array}{l}\text { Medication cost/co-pay } \\
\text { Poor access to medication } \\
\text { Poor aftercare/discharge planning } \\
\text { Poor physician-patient relationship } \\
\text { Poor physician communication }\end{array}$ \\
\hline
\end{tabular}

of 1,563 US and 614 European patients from three different countries (Belgium, the Netherlands, and Switzerland) were included. Prevalence of NA was higher in the US than in Europe (19.3\% vs $13.2 \%$ ), suggesting that the health-system care is a significant determinant of NA. ${ }^{20}$

Beliefs and satisfaction about medication are parameters that could predict adherence to immunosuppressants, and it has been proposed that beliefs about medication are more powerful predictors of reported adherence than clinical and sociodemographic factors. ${ }^{21}$ In renal transplantation, a link between negative beliefs on medication and NA has been established, ${ }^{22,23}$ suggesting that the implementation of psychological interventions, such as educational programs, cognitive-behavioral interventions, or counseling techniques may improve adherence to immunosuppressants. ${ }^{24}$

Depression has been found to increase the risk of treatment of NA threefold among patient populations. Both retrospective and prospective studies of kidney graft recipients report an association between NA and depression. ${ }^{25}$ In a recent study of kidney transplant patients, intentional NA (ie, choosing not to take them or skip/adjusting a dose) to immunosuppressant medications was associated with depression symptoms. ${ }^{26}$ However, other studies have failed to establish this link between depression and NA after adjusting for sociodemographic factors. ${ }^{27}$

One factor that has been linked to NA is the complexity of the treatment. For many chronic diseases, research has shown that adherence decreases as the complexity of the medication regimen increases (ie, the number of pills per dose and number of doses per day, the necessity to observe strict requirements related to the intake of food, and the existence of special requirements regarding fluid intake). ${ }^{1}$ The pharmaceutical industry has developed different medications for renal transplant patients aimed to simplify treatment and improve adherence. Noticeably, conflicting results were obtained with this approach. In the randomized multicenter controlled trial, to evaluate adherence between a tacrolimus once-daily regimen and a tacrolimus twice-daily regimen using an electronic monitor to document drug intake, ${ }^{28}$ patients allocated to the once-daily group take the prescribed number of daily doses more often than patients allocated to the twice-daily group ( $88.2 \%$ vs $78.8 \%$ ). However, the proportion of patients having at least 1-day interval without a dose per month (missing a single dose for once-daily regimen or missing two consecutive doses for twice-daily regimen) was higher for the once-daily group than for the twice-daily group (62\% vs 40\%). Similar results were also obtained in a recent observational study conducted in eight centers 
in France in which simple treatment regimens including a small number of doses per day and a small number of tablets per day were associated with NA during the first year. ${ }^{29} \mathrm{On}$ the contrary, in an small study $(n=76)$ conducted in stable heart transplant recipients, ${ }^{30}$ switching from conventional tacrolimus or cyclosporine (twice-daily dosing) to modifiedrelease tacrolimus (once-daily dosing) was associated with an improvement of adherence in $>50 \%$ of patients.

\section{Strategies to increase adherence to immunosuppressive treatment}

The WHO has classified barriers for adherence to medication in the following five main categories: ${ }^{1}$ a) socioeconomic factors (financial difficulties or lack of transportation), b) healthcare organization barriers (limited amount of time allocated to each patient or staff rotation), c) disease-related factors (depression and anxiety associated with chronic diseases), d) therapy-related factors (side effects of drugs, complex dose regimens), and e) patient-related factors (communication barriers, health attitudes, health beliefs, and health literacy). Thus, because patients have different barriers to medication adherence, it is important to implement an intervention that is tailored to their risk factors in order to improve their adherence rate.

It is beyond the scope of this paper to conduct a systematic review on interventional studies to improve adherence to immunosuppressant in renal transplant recipients. In 2009, a systematic review examined 12 intervention studies that have been conducted to improve medication adherence rates in solid organ transplant patients. ${ }^{31}$ Five reports focused on adult kidney transplant recipients and it was shown that multidimensional interventions are associated with improved medication adherence. Later on, in 2014, a systematic review aimed to assess the effectiveness of interventions to improve medication adherence in adult kidney transplantation identified 12 interventional studies. ${ }^{32}$ Once again, medication adherence rates were greatly enhanced when multidimensional interventions were implemented, whereas one-off feedback from a nurse and financial assistance programs offered little improvement. The clinical trial with the highest quality in this metaanalysis was conducted by Chisholm-Burns et $\mathrm{al}^{33}$ and showed that the multidimensional intervention, including informational (discussion about the consequences of NA), behavioral (identified routines, tools or strategies to increase adherence), and emotional (enhanced patient motivation) interventions, was associated with a higher adherence rate in the interventional than in the control group ( 0.89 vs 0.80$)$ and that this effect was maintained 3 months after completing the interventional period. Furthermore, this intervention was associated with a lower hospitalization rate $(24 \%$ vs $57 \%$ ). Finally, in a recent report, ${ }^{34}$ the implementation and evaluation of a multi-factorial intervention approach was tested using an educational program carried out in the form of intensified patient counseling by a dedicated clinical pharmacist. The additional pharmaceutical care was adjunct to an already established standardized basic drug and general transplant training program carried out by doctors and nurses. Combining educational, behavioral, and motivational interventions, the applied multifactorial intervention by the pharmaceutical care was associated with an improvement of drug adherence in the intervention group (91\% vs 75\%).

The economic impact of therapeutic NA in chronic diseases has rarely been examined. Long-term costs and outcomes in adherent and nonadherent renal transplant patients were simulated in a Markov model and, compared with dialysis; renal transplantation offers a better outcome in both adherent and nonadherent patients. ${ }^{35}$ Lifetime costs after transplantation in the adherent patient group are higher than lifetime dialysis costs and lifetime costs in the nonadherent patient group, mainly because adherent patients live longer after transplantation. The low incremental cost per quality-adjusted life-year (QALY) calculated in this model for adherent renal transplant patients suggests that there may be scope for adherence-enhancing interventions.

\section{Conclusion}

NA to immunosuppressant treatment is a common phenomenon after renal transplantation involving $>20 \%$ of patients and it is associated with the development of donor-specific antibodies and chronic rejection contributing to late graft failure in about $50 \%$ of failing grafts. Since the evaluation of sociodemographic factors do not allow characterizing the target population, it is necessary to combine different measures of adherence to increase its diagnostic accuracy. During the last decade, it has been shown that the implementation of a multidimensional intervention may lead to an improvement of adherence to treatment, whereas one-off feedback from a nurse, simplification of treatment, or financial assistance programs offered little improvement. Thus, increasing the effectiveness of adherence interventions might have a far greater impact on the long-term outcome of renal transplants than any improvement in specific medical treatments. This will require coordinated action from health professionals, researchers, health planners, and policy makers. 


\section{Author contributions}

All authors of the paper significantly contributed to 1) conception and design of data; 2 ) drafting the article or revising it critically for intellectual content; and 3) final approval of the version to be published.

\section{Acknowledgments}

This study was partly supported by a Fondo de Investigaciones Sanitarias (FIS) PI10/02496 and PIE13/00027, a Sociedad Española de Trasplante (SET), and a REDinREN $\mathrm{RD} / 0021 / 0013$ grants. IB Torres was supported by a Vall Hebron Research Institute (VHIR) grant.

\section{Disclosure}

The authors report no conflicts of interest in this work.

\section{References}

1. Sabaté E. Adherence to Long-Term Therapies. Evidence for Action. Switzerland: World Health organization; 2003.

2. Fine RN, Becker $\mathrm{Y}$, De Geest $\mathrm{S}$, et al. Nonadherence consensus conference summary report. Am J Transplant. 2009;9:35-41.

3. Wolfe RA, Ashby VB, Milford EL, et al. Comparison of mortality in all patients on dialysis, patients on dialysis awaiting transplantation, and recipients of a first cadaveric transplant. $N$ Engl J Med. 1999;341: 1725-1730.

4. Dew MA, DiMartini AF, De Vito Dabbs A, et al. Rates and risk factors for nonadherence to the medical regimen after adult solid organ transplantation. Transplantation. 2007;83:858-873.

5. Butler JA, Roderick P, Mullere M, Mason JC, Peveler RC. Frequency and impact of nonadherence to immunosuppressants after renal transplantation: a systematic review. Transplantation. 2004;77:769-789.

6. Hillbrands LB, Hoistma AJ, Koene RA. Medication compliance after renal transplantation. Transplantation. 1995;60:914-920.

7. Chisholm MA, Vollenweider LJ, Mulloy LL, et al. Renal transplant patient compliance with free immunosuppression. Transplantation. 2000;70:1240-1244.

8. Teixeira-De Barros C, Cabrita J. Noncompliance with immunosuppressive therapy: prevalence and determinants. Transplant Proc. 2000;32:2633.

9. Schäfer-Keller P, Steiger J, Bock A, Denhaerynck K, De Geest S. Diagnostic accuracy of measurement methods to assess non-adherence to immunosuppressive drugs in kidney transplant recipients. $\mathrm{Am} \mathrm{J}$ Transplant. 2008;8:616-626.

10. Morisky DE, Green LW, Levine DM. Concurrent and predictive validity of a self-reported measure of medication adherence. Med Care. 1986;24(1):67-74.

11. Chisholm MA, Lance CE, Williamson GM, Mulloy LL. Development and validation of the immunosuppressant therapy adherence instrument (ITAS). Patient Educ Couns. 2005;59(1):13-20.

12. Dobbels F, Lut B, De Geest S, et al; Transplant360 Task Force. The psychometric properties and practicability of self-report instruments to identify medication non-adherence in adult transplant patients to date: a systematic review. Transplantation. 2010;90(2):205-219.

13. De Geest S, Abraham I, Dunbar-Jacob J. Measuring transplant patients' compliance with immunosuppressive therapy. West $J$ Nurs Res. 1996;18(5):595-605.

14. de Oliveira Marsicano E, da Silva Fernandes N, Colugnati F, et al. Transcultural adaptation and initial validation of Brazilian-Portuguese version of the Basel assessment of adherence to immunosuppressive medications scale (BAASIS) in kidney transplants. BMC Nephrol. 2013;14:108.
15. Morris PJ. Transplantation - a medical miracle of the 20th century. N Engl J Med. 2004;351:2678-2680.

16. Nankivell BJ, Alexander SI. Rejection of the kidney allograft. $N$ Engl J Med. 2010;363:1451-1462.

17. Nevins TE, Kruse L, Skeans MA, Thomas W. The natural history of azathioprine compliance after renal transplantation. Kidney Int. 2001;60:1565-1570.

18. Sellarés J, de Freitas DG, Mengel M, et al. Understanding the causes of kidney transplant failure: the dominant role of antibody-mediated rejection and nonadherence. Am J Transplant. 2012;12:388-399.

19. Gaynor JJ, Ciancio G, Guerra G, et al. Graft failure due to noncompliance among 628 kidney transplant recipients with long-term follow-up: a single-center observational study. Transplantation. 2014;97:925-933.

20. Denhaerynck K, Desmyttere A, Dobbels F, et al. Nonadherence with immunosuppressive drugs: US compared with European kidney transplant recipients. Prog Transplant. 2006;16(3):206-214.

21. Horne R, Weinman J. Patients' beliefs about prescribed medicines and their role in adherence to treatment in chronic physical illness. J Psychosom Res. 1999;47:555-567.

22. Chisholm MA, Lance CE, Mulloy LL. Patient factors associated with adherence to immunosuppressant therapy in renal transplant recipients. Am J Health Syst Pharm. 2005;62(17):1775-1781.

23. Chisholm-Burns M, Pinsky B, Parker G, et al. Factors related to immunosuppressant medication adherence in renal transplant recipients. Clin Transplant. 2012;26:706-713.

24. Hugon A, Roustit M, Lehmann A, et al. Influence of intention to adhere, beliefs and satisfaction about medicines on adherence in solid organ transplant recipients. Transplantation. 2014;98:222-228.

25. Chilcot J, Spencer BWJ, Maple H, Mamode N. Depression and kidney transplantation. Transplantation. 2014;97:717-772.

26. Griva K, Davenport A, Harrison M, Newman SP. Non-adherence to immunosuppressive medications in kidney transplantation: intent vs forgetfulness and clinical markers of medication intake. Ann Behav Med. 2012;44:85.

27. Weng FL, Chandwani S, Kurtyka KM, Zacker C, Chisholm-Burns MA, Demissie K. Prevalence and correlates of medication non-adherence among kidney transplant recipients more than 6 months post-transplant: a cross-sectional study. BMC Nephrol. 2013;14:261-270.

28. Kuypers DR, Peeters PC, Sennesael JJ, et al; ADMIRAD Study Team. Improved adherence to tacrolimus once-daily formulation in renal recipients: a randomized controlled trial using electronic monitoring. Transplantation. 2013;95(2):333-340.

29. Couzi L, Moulin B, Morin MP, et al. Factors predictive of medication nonadherence after renal transplantation: a French observational study. Transplantation. 2013;95(2):326-332.

30. Doesch AO, Mueller S, Akyol C, et al. Increased adherence eight months after switch from twice daily calcineurin inhibitor based treatment to once daily modified released tacrolimus in heart transplantation. Drug Des Devel Ther. 2013;7:1253-1258.

31. De Bleser L, Matteson M, Dobbels F, Russell C, De Geest S. Interventions to improve medication-adherence: a systematic review. Transpl Int. 2009;22(8):780-797.

32. Low JK, Williams A, Manias E, Crawford K. Interventions to improve medication adherence in adult kidney transplant recipients: a systematic review. Nephrol Dial Transplant. 2014;pii:gfu204.

33. Chisholm-Burns MA, Spivey CA, Graff Zivin J, Lee JK, Sredzinski E, Tolley EA. Improving outcomes of renal transplant recipients with behavioral adherence contracts: a randomized controlled trial. $\mathrm{Am} \mathrm{J}$ Transplant. 2013;9:2364-2373.

34. Joost R, Dörje F, Schvitulla J, Eckardt KU, Hugo C. Intensified pharmaceutical care is improving immunosuppressive medication adherence in kidney transplant recipients during the first post-transplant year: a quasi-experimental study. Nephrol Dial Transplant. 2014;29(8): 1597-1607.

35. Cleemput I, Kesteloot K, Vanrenterghem Y, De Geest S. The economic implications of non-adherence after renal transplantation. Pharmacoeconomics. 2004;22(18):1217-1234. 


\section{Publish your work in this journal}

Transplant Research and Risk Management is an international, peerreviewed open access journal focusing on all aspects of transplantation and risk management to achieve optimal outcomes in the recipient improving survival and quality of life. The journal welcomes submitted papers covering original research, basic science, clinical studies,

reviews \& evaluations, guidelines, expert opinion and commentary, case reports and extended reports. The manuscript management system is completely online and includes a very quick and fair peer-review system, which is all easy to use. Visit http://www.dovepress.com/ testimonials.php to read real quotes from published authors.

Submit your manuscript here: http://www.dovepress.com/transplant-research-and-risk-management-journal 